FVDZ akademie

\title{
Seminar-Highlights im Juli
}

Die ausführliche Seminarübersicht finden Sie im Internet unter $\boldsymbol{w} \boldsymbol{w} \boldsymbol{w} . \boldsymbol{f} \boldsymbol{v} \boldsymbol{d} \boldsymbol{z} . \boldsymbol{d e}$.

\section{Betriebswirtschaft, Steuern und Finanzen}

Klassische Kooperationen, MVZ, Laborgemeinschaften, Zusammenarbeit mit Spezialisten - Wie positioniere ich meine Praxis?

(241) 09.07.16 Kassel Samstag 9.00-13.00 Uhr

Referenten: Dipl.-Kfm./Stb./WP Michael Laufenberg, Köln

RA Michael Lennartz, Bonn

Gebühren: Zahnärzte Mitglieder 195 EUR, Zahnärzte Nichtmitglieder 250 EUR, Ehepartner 150 EUR

\section{Praxismarketing und Kommunikation}

Geldgespräche mit Patienten in der Zahnarztpraxis

(342) 08.07.16 Nürnberg Freitag 14.00-20.00 Uhr

Referent: Dipl.-Oec. Hans Dieter Klein, Stuttgart

Gebühren: Zahnärzte Mitglieder 265 EUR, Zahnärzte Nichtmitglieder 320 EUR, Praxismitarbeiter/innen (Mitgliederpraxis) 215 EUR, Praxismitarbeiter/innen (Nichtmitgliederpraxis) 235 EUR

\section{Recht in der Zahnarztpraxis}

„Unwissen schützt vor Kürzung nicht" - Zahnärztliche Wirtschaftlichkeitsprüfung

(424) 09.07.16 Potsdam Samstag 10.00-14.00 Uhr

Referent: Dr. Dr. Klaus Oehler, Osnabrück

Gebühren: Zahnärzte Mitglieder 195 EUR, Zahnärzte Nichtmitglieder 250 EUR, Ehepartner 150 EUR
Fokus Zahnarztrecht 2016

NEU!

(442) 02.07.16 Dortmund Samstag 9.00-13.00 Uhr

Referenten: RA Dirk Wachendorf, Koblenz;

RA Michael Lennartz, Bonn - alternierend

Gebühren: Zahnärzte Mitglieder 195 EUR, Zahnärzte Nichtmitglieder 250 EUR

\section{Programm für Praxismitarbeiter/innen}

Moderne Psychologie in der Prophylaxe

Das Highlight-Seminar mit Tiefgang und vielen Raffinessen für das halbe Leben

(535) 01.07.16 München Freitag 14.00-20.00 Uhr

Referent: Dipl.-Päd. Herbert Prange, Bellavista - Mallorca

Gebühren: Praxismitarbeiter/innen (Mitgliederpraxis) 215 EUR, Praxismitarbeiter/innen (Nichtmitgliederpraxis) 235 EUR

\section{Sonderveranstaltungen}

Elegante Psychologie für die Praxis und das halbe Leben - Das Spezialseminar für den Chef und das ganze Team (604) 02.07.16 München Samstag 9.00-15.00 Uhr Referent: Dipl.-Päd. Herbert Prange, Bellavista - Mallorca

Gebühren: Zahnärzte Mitglieder 265 EUR, Zahnärzte Nichtmitglieder 320 EUR, Praxismitarbeiter/innen (Mitgliederpraxis) 215 EUR, Praxismitarbeiter/innen (Nichtmitgliederpraxis) 235 EUR

Bewertung der Fortbildungsveranstaltungen erfolgt nach dem gemeinsam von DGZMK/BZÄK verabschiedeten Punktesystem. 